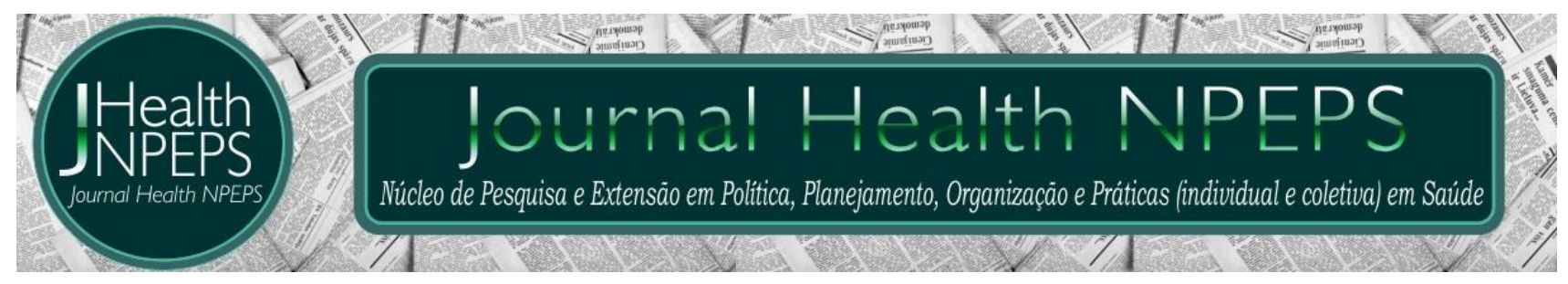

http://dx.doi.org/10.30681/252610104880

ARTIGO ORIGINAL

\title{
Cocos Gram-positivos resistentes isolados de profissionais de saúde e de ambiente hospitalar em Cuiabá-MT
}

\section{Gram-positive resistant cocci isolated from health professionals and hospital environment in Cuiabá-MT}

\section{Cocos Gram-positivos resistentes aislados de profesionales de salud y en ambiente hospitalario de Cuiabá-MT}

\author{
Alexandre Paulo Machado1, Marília Duarte Valim², Liliana Victorino Alves Corrêa ${ }^{3}$, \\ Emanuelle Righetto Corrêa ${ }^{4}$, Lais Rodrigues $\mathrm{Melo}^{5}$, Aline Tais Rothmund Topanotti ${ }^{6}$, \\ Vitor Toshio Atakiama7, Marco Andrey Pepato ${ }^{8}$
}

\begin{abstract}
RESUMO
Objetivo: caracterizar cocos Gram-positivos resistentes a antimicrobianos presentes nas mãos de profissionais de saúde e em ambiente hospitalar. Método: estudo transversal e descritivo, com coleta de amostras das mãos de profissionais de saúde e nas superfícies das unidades de terapia semi-intensiva e intensiva, em um hospital universitário de Cuiabá, Mato Grosso, Brasil. As bactérias foram isoladas em meios seletivos contendo oxacilina e identificadas por Vitek ${ }^{\circledR} 2$, a partir dos perfis bioquímicos e de suscetibilidade aos antibióticos. Resultados: dos 63 isolados analisados, 56 (88,9\%) pertenciam ao gênero Staphylococcus e sete $(11,1 \%)$ ao gênero Enterococcus. Destes, 44 foram provenientes de amostras das mãos dos profissionais de saúde e 19 do ambiente hospitalar. Staphylococcus epidermidis e Staphylococcus haemolyticus foram as espécies
\end{abstract}

\footnotetext{
${ }^{1}$ Biólogo. Doutor em Ciências. Professor Associado do Departamento de Ciências Básicas da Saúde, da Faculdade de Medicina, da Universidade Federal de Mato Grosso. Cuiabá, Mato Grosso, Brasil. E-mail: alepaulo@hotmail.com ORCID ID: https://orcid.org/0000-0002-1342-8132 Autor para correspondência - Endereço: Faculdade de Medicina, Av. Fernando Corrêa da Costa, no 2367, Bairro Boa Esperança, Cuiabá/MT, CEP: 78060-900.

${ }^{2}$ Enfermeira. Doutora em Ciências da Saúde. Professora Adjunta na Universidade Federal do Mato Grosso (UFMT). Cuiabá, Mato Grosso, Brasil. E-mail: marilia.duarte.valim@gmail.com ORCID ID: https://orcid.org/0000-0002-2746-1865

${ }^{3}$ Biólogo. Mestre em Engenharia Ambiental. Cuiabá, Mato Grosso, Brasil. E-mail: licke bio@hotmail.com ORCID ID: https://orcid.org/0000-0002-8897-2183

${ }^{4}$ Enfermeira. Especialização em Enfermagem em Unidade de Terapia Intensiva. Faculdade de Medicina, Cuiabá, Mato Grosso, Brasil. E-mail: emanuellerighetto@gmail.com ORCID ID: https://orcid.org/0000-0002-2810-7120

${ }^{5}$ Graduanda da Faculdade de Medicina da UFMT. Cuiabá, Mato Grosso, Brasil. E-mail: lais rodmelo@hotmail.com ORCID ID: https://orcid.org/0000-0003-0140-1298

${ }^{6}$ Graduanda da Faculdade de Medicina da UFMT. Cuiabá, Mato Grosso, Brasil. E-mail: alinetopanotti15@gmail.com ORCID ID: https://orcid.org/0000-0003-2691-3038

${ }^{7}$ Graduando da Faculdade de Medicina da UFMT. Cuiabá, Mato Grosso, Brasil. E-mail: vitortoshio@gmail.com ORCID ID: https://orcid.org/0000-0003-1053-7445

${ }^{8}$ Farmacêutico. Doutor. Servidor do Governo do Estado do Mato Grosso e da Universidade Federal de Mato Grosso. Cuiabá, Mato Grosso, Brasil. E-mail: mcoandrey@gmail.com ORCID ID: $\underline{\text { https://orcid.org/0000-0002-7896-6329 }}$
}

Este artigo está licenciado sob forma de uma licença Creative Commons Atribuição 4.0 Internacional, que permite uso irrestrito, distribuição e reprodução em qualquer meio, desde que a publicação original seja corretamente citada. 
mais prevalentes. Perfil multirresistente foi verificado em 61 isolados $(96,8 \%)$. Em 47 Staphylococcus spp. com indicação de modificação de PBP, detectou-se 42 fenótipos sugestivos de MLSB constitutivos e cinco induzíveis. Um isolado de amostra da mão era Enterococcus faecalis perfil VanA. Conclusão: destaca-se a importância da correta higiene das mãos e descontaminação do ambiente hospitalar, uma vez que foram encontrados cocos Gram-positivos principalmente multirresistentes em superfícies bióticas e abióticas.

Descritores: Cocos Gram-Positivos; Farmacorresistência Bacteriana; Infecção Hospitalar.

\section{ABSTRACT}

Objective: to characterize gram-positive cocci resistant to antimicrobials present in the hands of health professionals and in the hospital environment. Method: crosssectional and descriptive study, with collection of samples from the hands of health professionals and on the surfaces of semi-intensive and intensive care units, in $a$ university hospital in Cuiabá, Mato Grosso, Brazil. The bacteria were isolated in selective media containing oxacillin and identified by Vitek ${ }^{\circledR} 2$, based on biochemical profiles and susceptibility to antibiotics. Results: of the 63 isolates analyzed, 56 (88.9\%) belonged to the Staphylococcus genus and seven (11.1\%) to the Enterococcus genus. Of these, 44 came from samples of the hands of health professionals and 19 from the hospital environment. Staphylococcus epidermidis and Staphylococcus haemolyticus were the most prevalent species. Multidrug resistant profile was verified in 61 isolates (96.8\%). In 47 Staphylococcus spp. with indication of PBP modification, 42 constitutive and five inducible MLSB phenotypes were detected. A sample isolate from the hand was Enterococcus faecalis VanA profile. Conclusion: the importance of correct hand hygiene and decontamination of the hospital environment is highlighted, since gram-positive cocci were found, mainly multiresistant, on biotic and abiotic surfaces.

Descriptors: Gram-Positive Cocci; Bacterial Drug Resistance; Hospital Infection.

\section{RESUMEN}

Objetivo: caracterizar cocos Gram-positivos resistentes en las manos de los profesionales de salud y en el entorno hospitalario. Método: Estudio transversal y descriptivo, con recolección de muestras de manos de profesionales de la salud y en las superficies de unidades de cuidados intensivos y semi-intensivos, en un hospital universitario de Cuiabá, Mato Grosso, Brasil. Las bacterias se aislaron en medios selectivos que contenían oxacilina y se identificaron mediante Vitek®, 2 a partir de los perfiles bioquímicos y la susceptibilidad a los antibióticos. Resultados: de los 63 aislamientos analizados, 56 (88,9\%) pertenecían al género Staphylococcus y siete $(11,1 \%)$ al género Enterococcus. De estos, 44 procedian de muestras de manos de profesionales sanitarios y 19 del ámbito hospitalario. Staphylococcus epidermidis y Staphylococcus haemolyticus fueron las especies más prevalentes. El perfil de multirresistencia se verificó en 61 aislamientos (96,8\%). En 47 Staphylococcus spp. con indicación de modificación de PBP, se detectaron 42 fenotipos MLSB constitutivos y cinco inducibles. Una muestra aislada de la mano fue Enterococcus faecalis perfil VanA. Conclusión: se destaca la importancia de una correcta higiene de manos y descontaminación del ambiente hospitalario, ya que se encontraron cocos grampositivos, principalmente multirresistentes, sobre superficies bióticas y abióticas.

Descriptores: Cocos Grampositivos; Farmacorresistencia Bacteriana; Infección Hospitalaria. 


\section{INTRODUÇÃO}

As infecções relacionadas à assistência à saúde (IRAS) são responsáveis por elevar as taxas de morbimortalidade, altos custos para o Sistema Único de Saúde (SUS) e tempo prolongado de internação. Sabe-se que a maior parte dos patógenos que causa as infecções hospitalares estão presentes na microbiota dos pacientes, dos profissionais de saúde (principalmente nas mãos) e no ambiente hospitalar ${ }^{1,2}$. Ainda, observa-se que o tempo prolongado de internação está diretamente relacionado com o aumento das taxas de IRAS. Nesse sentido, a baixa adesão a lavagem das mãos pelos profissionais de saúde, bem como a higienização incorreta são as principais causas da disseminação desses patógenos no ambiente hospitalar ${ }^{1}$.

Segundo o European Center for Disease Prevention and Control, 20 a $30 \%$ das IRAS são consideradas preveníveis por meio de higienização do ambiente e das mãos dos profissionais de saúde, bem como uma estrutura hospitalar adequada segundo as normas do país².

Ademais, as IRAS têm relação direta com o processo de evolução da resistência bacteriana antimicrobianos devido à pressão seletiva gerada pelo uso (inclusive incorreto) de antibióticos e desinfetantes, seja pela população ou pela assistência à saúde ${ }^{2,3}$. Nesse contexto, as IRAS causadas por bactérias carreando genes de resistência aos antimicrobianos e de virulência são ainda mais danosas e preocupantes, visto que o tratamento ocorre por um maior período de tempo, além de ter maior taxa de falha relacionada ao incremento de óbitos, principalmente em indivíduos imunocomprometidos ou com doenças crônicas².

Os cocos Gram-positivos são microrganismos muito frequentemente isolados em amostras biológicas humanas e de animais com amplos mecanismos de resistência aos antimicrobianos ${ }^{1,3}$. Destes, os principais patógenos relacionados às IRAS correspondem aos gêneros Staphylococcus e Enterococcus. A multirresistência aos antibióticos e a sua dispersão são fenômenos que têm preocupado as autoridades sanitárias pelo mundo. Nesse sentido, o objetivo desse estudo foi caracterizar cocos Gram-positivos resistentes a antimicrobianos presentes nas mãos de profissionais de saúde e em ambiente hospitalar. 


\section{MÉTODO}

Estudo transversal e descritivo, realizado a partir da coleta de amostras das mãos de profissionais da saúde e do ambiente onde trabalhavam em um hospital universitário de referência em Cuiabá - Mato Grosso, na ala da Clínica Médica, Unidade Semi-Intensivo Adulto e Unidade de Terapia Intensiva Adulto (UTI) entre os meses de fevereiro a junho de 2018.

A coleta foi realizada com trabalhadores da equipe de enfermagem (enfermeiros, técnicos de enfermagem e auxiliares) que representaram os profissionais de saúde, durante o plantão. Nesse espaço amostral havia 74 profissionais de saúde, sendo escolhidos por representar a maioria na equipe de atenção à saúde e por estarem em contato direto mais frequentemente com os pacientes. Foi utilizado como critério de inclusão: profissionais de enfermagem que executassem atividades assistenciais de cuidado aos pacientes. Foram excluídos profissionais que exerciam atividades exclusivamente administrativas e que fizeram uso de agentes esteroides no último mês, a fim de que a microbiota das mãos dos profissionais seja avaliada em condições similares. Excluíram-se também, profissionais que apresentaram mãos com integridade da pele prejudicada com ferimento, eczema e/ou psoríase, ou outras lesões de pele relevantes.

Destes profissionais de saúde, haviam 25 técnicos de enfermagem e sete enfermeiros em UTI adulto. Já os conjuntos da ala de clínica médica/unidade semi-intensiva adulto foi representado por nove auxiliares de enfermagem, 23 técnicos de enfermagem e 10 enfermeiros.

Para coleta da microbiota presente nas mãos solicitou-se que os profissionais inserissem a mão dominante em um saco plástico contendo $200 \mathrm{~mL}$ de infusão cérebro e coração previamente esterilizado, sendo massageada por 1 minuto contra a superfície do saco plástico.

0 total de 40 amostras de superfícies inanimadas (20 presentes na clínica médica e 20 na UTI) foram coletadas de objetos e de dispositivos utilizados diariamente na rotina de assistência em saúde para avaliação da microbiota presente nesses potenciais fômites, sendo provenientes de teclados (3), maçanetas (4), telefone (2), bomba de infusão (5), monitores multiparâmetros (2), mesa de cabeceira (1), grade lateral do leito (4), chão (5), torneira (2), glicosímetro (1), capa de 
prontuário (1), tampa de lixeira (2), frasco de dieta (1), frasco de aspiração (1), dispensador de sabonete (1), mouse de computador (2) e apoio braço da cadeira (1) e bancada de preparo de medicação (2). Os locais selecionados e a hora de coleta de amostras não foram informados aos profissionais de saúde para reprodução fidedigna do cenário avaliado. Amostras de ambas as superfícies (mãos e fômites) eram coletadas por visita, aleatoriamente, nos locais de estudo.

Para coleta foram utilizados swabs esterilizados de algodão e umedecidos com solução salina para friccionar a superfície de objetos em zigue-zague por única vez, sendo imersos em tubos com tampa contendo $2 \mathrm{~mL}$ de meio BHI (Brain Heart Infusion Broth, Oxoid), identificados e enviados imediatamente para análise no laboratório em caixas térmicas com gelo sob conservação de 4 a $8^{\circ} \mathrm{C}$.

Antes da semeadura, as amostras eram homogeneizadas em agitador vortex analógico (QL-901, Eikonal do Brasil) e, seguidamente, aplicadas em ágar Mueller-Hinton suplementado com $4 \% \mathrm{NaCl}$ e $6 \mu \mathrm{g} / \mathrm{mL}$ de oxacilina (Thermofisher Scientific), pelos métodos de espalhamento em superfície (spread plate) para os espécimes provenientes das mãos e estriamento em zigue-zague para aqueles de origem ambiental, sendo as culturas incubadas em estufa microbiológica à $35^{\circ} \mathrm{C}$ por até 72 horas. Três colônias com morfotipos semelhantes eram selecionadas para confirmação da identidade microbiana. A partir de culturas puras, cartões para identificação de bactérias Grampositivas foram inoculados e analisados no equipamento Vitek ${ }^{\circledR} \quad 2$ para identificar os microrganismos conforme o perfil bioquímico e de resistência aos antimicrobianos, conforme instruções do fabricante. A identidade dos isolados ao nível de gênero e de espécie foi confirmada também por Maldi-Tof (Maldi Biotyper, Bruker DaltoniK).

Este estudo teve projeto de pesquisa submetido ao Comitê de Ética em Pesquisa do Hospital Universitário Júlio Muller conforme resolução $\mathrm{n}$. 466/2012 do Conselho Nacional de Saúde e aprovado com o número de Certificado de Apresentação para Apreciação Ética (CAAE) n. 75169317.0.0000.5541. Além disso, foram obtidas autorizações da administração da UTI adulto, da Clínica Médica e da Unidade de Semi-Intensivo Adulto e da Gerência de Educação e Pesquisa (GEP), Serviço de Controle de Infecção Hospitalar (SCIH) e Núcleo de Segurança do Paciente. 
RESULTADOS

Das 74 amostras colhidas a partir das superfícies de mãos dos profissionais de saúde e das 40 de objetos do ambiente hospitalar, isolou-se 63 cocos Gram-positivos, sendo 56 do gênero Staphylococcus e sete do gênero Enterococcus. Do total de cocos Grampositivos identificados, $44 \quad(69,84 \%)$ provinham de amostras das mãos dos profissionais de saúde colhidas durante o expediente e 19 (30,16\%) do ambiente hospitalar. A Tabela 1 detalha as espécies detectadas conforme suas respectivas superfícies. Verificou-se maior prevalência de Staphylococcus epidermidis, Staphylococcus haemolyticus e Enterococcus faecalis, em especial, nas mãos.

\begin{tabular}{|c|c|c|c|}
\hline Espécie & $\begin{array}{l}\text { Mãos } \\
\text { n (\%) }\end{array}$ & $\begin{array}{c}\text { Ambiente } \\
\text { n (\%) }\end{array}$ & $\begin{array}{l}\text { Total } \\
\text { n (\%) }\end{array}$ \\
\hline Staphyloccocus aureus & $1(2,27)$ & $2(10,53)$ & $3(4,76)$ \\
\hline Staphylococcus capitis & 0 & $1(5,26)$ & $1(1,59)$ \\
\hline Staphylococcus epidermidis & $13(29,55)$ & $2(10,53)$ & $15(23,81)$ \\
\hline Staphylococcus haemolyticus & $21(47,73)$ & $6(31,58)$ & $27(42,86)$ \\
\hline Staphylococcus hominis ssp hominis & 0 & $3(15,79)$ & $3(4,76)$ \\
\hline Staphylococcus lentus & 0 & $1(5,26)$ & $1(1,59)$ \\
\hline Staphylococcus saprophyticus & $1(2,27)$ & $2(10,53)$ & $3(4,76)$ \\
\hline Staphylococcus warneri & $2(4,55)$ & 0 & $2(3,17)$ \\
\hline Enterococcus faecalis & $5(11,36)$ & 0 & $5(7,94)$ \\
\hline Enterococcus faecium & $1(2,27)$ & $1(5,26)$ & $2(3,17)$ \\
\hline Enterococcus hirae & 0 & $1(5,26)$ & $1(1,59)$ \\
\hline Total & $44(69,84)$ & $19(30,16)$ & $63(100)$ \\
\hline
\end{tabular}

O perfil fenotípico de resistência dos cocos Gram-positivos isolados a determinados antibióticos encontram-se dispostos na tabela 2. $\mathrm{Na}$ análise automatizada no equipamento VITEK 2 revelou indicação sugestiva da ocorrência de modificação da proteína ligadora à penicilina (PBP, sigla do inglês) em 51 isolados (37 das mãos/14 do ambiente hospitalar) do gênero Staphylococcus. Destes fenótipos MecA, foi verificado compatibilidade com perfil de resistência à macrolídeos, lincosaminas e estreptogramina B (MLSB, sigla do inglês), sendo 42 constitutivos e 5 induzíveis. Destes, os perfis sugestivos de MLSB constitutivos foram: $01 \mathrm{~S}$. aureus (ambiente), $01 \quad$ S. capitis 
(ambiente), 10 S. epidermidis (09 das mãos/01 do ambiente), $26 \mathrm{~S}$. haemolyticus (20 das mãos/06 do ambiente), $01 \mathrm{~S}$. hominis ssp hominis (ambiente), $01 \quad$ S. saprophyticus (ambiente) e 02 S. warneri (mãos). Enquanto os isolados com fenótipo induzivel foram: 02 S. epidermidis (mãos), 01 S. haemolyticus (mão) e $02 \mathrm{~S}$. saprophyticus (mão/ambiente). Menor susceptibilidade para o glicopeptídeo teicoplanina também foi observada para dois isolados MecA e para 16 com perfil MecA-MLSB.

Tabela 2 - Fenótipos de mono e multirresistência à antibióticos de 63 isolados de cocos Gram-positivos isolados de superfícies de objetos presentes no ambiente hospitalar e das mãos de profissionais de saúde em hospital universitário. Cuiabá (MT), Brasil.

\begin{tabular}{|c|c|c|c|c|}
\hline Espécies & Fenótipos de resistência & Superfícies & $\mathrm{n}$ & $\%$ \\
\hline \multirow[t]{2}{*}{ Staphylococcus aureus } & PEN-OXA & $M / A$ & 2 & 66,6 \\
\hline & CFO-PEN-GEN-LVX-ERI-CLI & $A$ & 1 & 33,3 \\
\hline S. capitis & CFO-PEN-OXA-GEN-LVX-ERI-CLI & A & 1 & 100,0 \\
\hline \multirow[t]{11}{*}{ S. epidermidis } & CFO-PEN-OXA & M & 1 & 6,7 \\
\hline & CFO-PEN-OXA-ERI-CLI & M & 1 & 6,7 \\
\hline & CFO-PEN-OXA-ERI-CLI-RIF & M & 2 & 13,3 \\
\hline & CFO-PEN-OXA-ERI-CLI-TSU & M & 1 & 6,7 \\
\hline & CFO-PEN-OXA-GEN-ERI-CLI & M & 1 & 6,7 \\
\hline & CFO-PEN-OXA-GEN-LVX-ERI-CLI-RIF & $A$ & 1 & 6,7 \\
\hline & CFO-PEN-OXA-GEN-LVX-RIF-TSU & A & 1 & 6,7 \\
\hline & CFO-PEN-OXA-LVX & M & 1 & 6,7 \\
\hline & CFO-PEN-OXA-LVX-ERI-CLI & M & 1 & 6,7 \\
\hline & CFO-PEN-OXA-LVX-ERI-CLI-RIF & M & 4 & 26,7 \\
\hline & CFO-PEN-OXA-LVX-ERI-CLI-TSU & M & 1 & 6,7 \\
\hline \multirow[t]{6}{*}{ S. haemolyticus } & CFO-PEN-OXA-RIC-ERI-CLI & M & 1 & 3,7 \\
\hline & CFO-PEN-OXA-LVX-ERI-CLI-RIF-TSU & M & 1 & 3,7 \\
\hline & CFO-PEN-OXA-GEN-LVX-ERI-CLI & $2 \mathrm{~A} / 2 \mathrm{M}$ & 4 & 14,8 \\
\hline & CFO-PEN-OXA-GEN-LVX-ERI-CLI-RIF & M & 6 & 22,2 \\
\hline & CFO-PEN-OXA-GEN-LVX-ERI-CLI-TSU & $2 \mathrm{~A} / 4 \mathrm{M}$ & 6 & 22,2 \\
\hline & CFO-PEN-OXA-GEN-LVX-ERI-CLI-RIF-TSU & $2 \mathrm{~A} / 7 \mathrm{M}$ & 9 & 33,3 \\
\hline \multirow[t]{3}{*}{ S. hominis ssp hominis } & PEN-OXA & A & 1 & 33,3 \\
\hline & PEN-ERI & A & 1 & 33,3 \\
\hline & CFO-PEN-OXA-LVX-ERI-CLI & A & 1 & 33,3 \\
\hline S. lentus & CFO-PEN-OXA-LVX-ERI-CLI-RIF-TSU & A & 1 & 100,0 \\
\hline \multirow[t]{3}{*}{ S. saprophyticus } & PEN-ERI-CLI & A & 1 & 33,3 \\
\hline & PEN-RIC-ERI-CLI & A & 1 & 33,3 \\
\hline & CFO-PEN-OXA-RIC-ERI-CLI-TSU & M & 1 & 33,3 \\
\hline S. warneri & CFO-PEN-OXA-ERI-CLI-RIF & M & 2 & 100,0 \\
\hline Enterococcus faecalis & ERI-CLI-TSU & M & 2 & 40,0 \\
\hline
\end{tabular}




\begin{tabular}{llccc} 
& EAN-ERI-CLI-TSU & $M$ & 2 & 40,0 \\
& GEN-EAN-LVX-ERI-CLI-TEC-VAN-TSU* & $M$ & 1 & 20,0 \\
\hline E. faecium & AMP-EAN-LVX-ERI-CLI-NIT-TSU & $\mathrm{A}$ & 1 & 100,0 \\
\hline E. hirae & CLI-NIT & $\mathrm{A}$ & 1 & 100,0
\end{tabular}

CFO: cefoxitina, PEN: benzilpenicilina, AMP: ampicilina, OXA: oxacilina, GEN: gentamicina, LVX: levofloxacina, CLI: clindamicina, EAN: estreptomicina alto nível, ERI: eritromicina, RIF: rifampicina, TEC: Teicoplanina, TSU: trimetropim/sulfametoxazol, VAN: vancomicina, NIT: nitrofurantoína. A: ambiente, M: mão.* Perfil Van A isolado das mãos de profissional da saúde.

\section{DISCUSSÃO}

A introdução dos antibióticos na prática clínica trouxe inúmeros benefícios, em especial, o controle das infecções que antes possuíam alta taxa de mortalidade. No entanto, o uso indiscriminado, hospitalar e agropecuário, fez com que o processo de resistência bacteriana fosse acelerado e tornou-se um grande problema de saúde pública $^{1,4}$. Muitas vezes o uso incorreto do medicamento pode acontecer por uma dosagem insuficiente, pela duração do tratamento menor do que 0 recomendado ou pelo uso inadequado para tratar outras doenças que não respondem a antimicrobianos como acontece na automedicação ${ }^{5,6}$.

A resistência bacteriana é definida como a capacidade da bactéria de sobreviver a uma determinada concentração de antimicrobianos que inibem o crescimento ou matam as bactérias de uma mesma espécie ${ }^{1,4}$. Esse processo ocorre devido à pressão seletiva exercida pelo antimicrobiano sobre as células microbianas que se modificaram, em exemplo, devido à indução da expressão gênica, mutação ou recepção de genes codificadores de resistência por transferência horizontal 5,6. Assim, as bactérias menos resistentes são eliminadas e as com genes de resistência podem sobreviver e causar infecções de difícil controle ${ }^{1}$. A aquisição de resistência cruzada é outro mecanismo importante, em particular, o uso de antimicrobianos na produção animal similares aos que são utilizados na antibioticoterapia humana, como no caso da avoparcina e vancomicina, respectivamente. Ela era utilizada na União Europeia, onde verificou-se um aumento de enterococos resistentes à vancomicina. Após a abolição do seu uso, notou-se diminuição do nível de resistência7 .

Nesse cenário de contribuição ao aumento de resistência bacteriana destacam-se as IRAS, que são definidas 
como infecções decorrentes do processo de cuidado em um hospital ou unidade prestadora de assistência à saúde não manifestadas na admissão do paciente ${ }^{1}$. Além disso, no ambiente hospitalar muitos pacientes estão imunossuprimidos e acabam desenvolvendo a doença e agravando 0 quadro ${ }^{5}$. As bactérias causadoras dessas infecções podem apresentar resistência a múltiplos agentes biocidas, tais como antibióticos e desinfetantes, uma vez que estão em um ambiente de maior pressão seletiva e, às vezes, até por falta de cuidados sanitários e de higiene adequados ${ }^{8}$.

Os agentes resistentes podem ter origem comunitária, ambiental ou hospitalar' ${ }^{9}$ Superfícies animadas e inanimadas foram avaliadas nesse trabalho a partir de amostras oriundas das mãos de profissionais da saúde e do ambiente hospitalar. Utilizando meio seletivo, avaliou a prevalência de cocos Gram-positivos resistentes à oxacilina, sendo detectado a presença de espécies dos gêneros Staphylococcus $e$ Enterococcus.

Os estafilococos associados à microbiota humana são patógenos oportunistas ubiquitários, como as espécies S. aureus, S. epidermidis, S. haemolyticus, S. saprophyticus, S. capitis, S. hominis e S. warneri comumente encontradas em amostras clínicas $^{9,10}$. A espécie $S$. aureus apresentou cepas produtoras de penicilinases na década de 50 , o que levou ao uso da meticilina. Porém, já em 1960 foram isoladas amostras resistentes a meticilina (MRSA) ${ }^{11}$. Dados do National Nososcomical Infections Surveillance (NNIS) mostram que a partir da década de 90 , cepas de $S$. aureus resistentes à meticilina ultrapassavam 50\% dos pacientes em UTII ${ }^{12}$ Os resultados fenotípicos dos isolados de S. aureus analisados na pesquisa demonstraram resistência à oxacilina pelo equipamento ViteK II, os quais se denominam de $S$. aureus resistentes à oxacilina (ORSA) ou MRSA, visto que a oxacilina é uma penicilina resistente às $B$-lactamases, assim como a meticilina. Portanto, os principais mecanismos de resistência microbiana esperados para as espécies de estafilococos, com base na metodologia de seleção empregada nessa pesquisa, seriam alteração de alvo e bombas de efluxo. A avaliação automatizada pelo equipamento Vitek II sugeriu perfil MecA (modificação de PBP) para a maioria dos isolados, todavia não se exclui os mecanismos de extrusão multidrogas pois fenótipos compatíveis como MSLB foram também indicados. 
Não obstante, a maior prevalência foi das espécies de Staphylococcus coagulase-negativa $(\mathrm{SCN})$, principalmente com perfil fenotípico resistente à oxacilina, bem como resistência a outras penicilinas e a outros antimicrobianos como a eritromicina, clindamicina, trimetropim/sulfametoxazol e a rifampicina que são utilizados para tratamentos de infecções bacterianas ${ }^{11}$.

Em comparação à transmissão de S. aureus resistente à meticilina (MRSA), se sabe menos sobre a epidemiologia dos SCN em unidades de saúde, bem como do seu potencial para causar surtos ${ }^{9}$. Estudo em hospital terciário na Tailândia demonstrou maior índice das espécies $S$. haemolyticus, S. epidermidis, S. saprophyticus, S. hominis em amostras clínicas. Dos 229 isolados de SCN resistentes à meticilina, 145 (63,32\%) continham 0 gene $\operatorname{MecA}^{13}$. Uma investigação dos genótipos de isolados nosocomiais de Staphylococcus epidermidis resistentes à meticilina revelou que profissionais de saúde eram portadores nasais (89\%) desse perfil e a colonização nos pacientes da comunidade ocorria, após a admissão, dentro de três dias, durante a hospitalização ${ }^{14}$.
Os

quimioterápicos

antimicrobianos, incluindo os Blactâmicos, têm se tornado progressivamente ineficientes frente aos isolados clínicos de CoNS por consequência da contínua aquisição de resistência e pressão seletiva9 . Esta situação é ainda mais agravada pelo fenômeno da multirresistência. No presente trabalho, as espécies $S$. epidermidis e S. haemolyticus foram as mais prevalentes e, em sua maioria, multirresistentes. Essas duas espécies também foram as mais prevalentes em hospital escola na cidade de Santa Maria, Estado do Rio Grande do Sul ${ }^{15}$. São patógenos oportunistas comuns em IRAS, difíceis de tratar e formadoras de biofilme ${ }^{16,17}$.

No tratamento de pioartrite, osteomielite e em infecções de fraturas expostas causadas por S. aureus e outras espécies do mesmo gênero, os principais antimicrobianos utilizados são a oxacilina, ciprofloxacina e cefalosporinas de primeira geração ${ }^{18}$. Contudo, bactérias MRSA possuem resistência a todos os antimicrobianos betalactâmicos, sendo a alternativa terapêutica nesses casos os medicamentos não beta-lactâmicos, como macrolídeos ou lincosamidas ${ }^{19}$. 
Todos os cocos Gram-positivos detectados neste estudo foram resistentes à oxacilina e a maioria destes isolados tinha perfil multirresistente, em especial, com fenótipos MLSB. Isso representa um achado preocupante, uma vez que esse tipo de fenótipo proporciona resistência aos grupos de antimicrobianos macrolídeo-lincosamidaestreptogramina B, que, apesar de serem estruturalmente diferentes entre $\mathrm{si}$, tem ação semelhante como não betalactâmicos ${ }^{19}$. Desse modo, apesar de ser um achado importante, já bem documentado em pesquisas internacionais $^{19,20,21}$, nota-se ainda no Estado de Mato Grosso uma carência de estudos sobre esse tipo de resistência.

Como algumas espécies de SCN têm tendência a resistência múltipla aos antibióticos e como muitas amostras indicaram a sua presença, a descrição é relevante pois podem se tornar um sério problema de saúde pública. Bactérias podem compartilhar, intra e interespecificamente, características que as aproximam nas vantagens adaptativas, especialmente sob condições de estresse ambiental, conferindo aos receptores uma menor susceptibilidade às intemperes do meio e, consequentemente, maior sobrevida e sucesso evolutivo. Isolados hospitalares de S. epidermidis e S. aureus transferem entre si não apenas loci de resistência aos antimicrobianos, mas também genes envolvidos na patogenicidade e resistência à toxicidade de metais ${ }^{22}$.

Com relação ao gênero Enterococcus, atualmente as espécies deste grupo causam infecções de difícil tratamento, incluindo endocardite infecciosa, sendo que a estratégia de tratamento antimicrobiano mais utilizada engloba o uso de: ampicilina, penicilina cristalina e vancomicina ${ }^{23}$. Em anos anteriores, esse gênero apresentava baixa virulência e causava repercussão clínica apenas em pacientes imunossuprimidos. No entanto, a partir da década de 80 passaram a ser identificadas cepas resistentes à vancomicina, fazendo com que as implicações clínicas e a taxa de mortalidade aumentassem exponencialmente ${ }^{24}$.

Sabe-se atualmente que são fatores de risco para emergência de Enterococcus resistentes à vancomicina: hospitalização prolongada em UTI, idade jovem e uso de certos quimioterápicos antimicrobianos como ceftriaxone e vancomicina ${ }^{25}$. No entanto, essas bactérias também podem ser transmitidas por meio das mãos dos profissionais de saúde, visto que 
sobrevivem por até 30 minutos após a devida lavagem destas ${ }^{26}$. Duas espécies causam a maioria das infecções enterocócicas: Enterococcus faecalis e Enterococcus faecium, sendo a primeira mais prevalente dentre os isolados clínicos $^{27}$. Nessa investigação, foi detectada prevalência nas mãos de profissionais de saúde da espécie Enterococcus faecalis. Em geral, isolados de $E$. faecium são mais virulentos e tem maior propensão para resistência aos antimicrobianos, incluindo a vancomicina $^{28,29}$. Considerando isto, um isolado de Enterococcus faecalis da mão de profissional da saúde analisado nesta pesquisa demonstrou ser perfil Van-A, o que denota relevância pois o gene VanA propicia a expressão de resistência à vancomicina, sendo que sua ocorrência pode estar paradoxalmente relacionada ao uso da própria vancomicina no tratamento de infecções ${ }^{30}$ ou ter origem animal devido à pressão seletiva com a utilização de antibióticos similares na pecuária $^{7,31}$. As observações se mostraram importantes, pois a multirresistência em enterococos está se intensificando nos últimos anos e o padrão de frequência desses patógenos como colonizadores de superfícies ou agentes infecciosos em hospitais vem se alterando gradativamente de acordo com a sucessiva inclusão de novos antimicrobianos na assistência em saúde.

Por fim, a maior parte dos isolados das mãos da equipe profissional de saúde e das superfícies de ambiente hospitalar analisados revelou perfis fenotípicos de multirresistência aos antimicrobianos comumente utilizados no tratamento das infecções. A alta prevalência de Staphylococcus spp e Enterococcus spp multirresistentes denota a necessidade institucional de compreender melhor a epidemiologia das IRAs e a correlação destas com os fenótipos encontrados, bem como intensificar os esforços de vigilância e controle destes agentes devido a sua importância como patógenos causadores de infecção nosocomial.

A limitação deste estudo consistiu em não realizar análises moleculares dos isolados, o que possivelmente acrescentaria mais informações sobre os perfis multirresistentes e sua dispersão no ambiente hospitalar.

\section{CONCLUSÃO}

Cocos Gram-positivos provenientes de amostras das mãos de profissionais de saúde e de superfícies inanimadas no ambiente hospitalar foram isolados a 
partir de meio seletivo contendo oxacilina, sendo identificadas espécies dos gêneros Staphylococcus e Enterococcus. Perfis fenótipos de multirresistência aos antimicrobianos foram mais prevalentes, o que é preocupante, pois tais traços vêm sendo associados à ineficácia de tratamento com os antibióticos mais amplamente difundidos e eficientes nos casos de infecções por essas bactérias, incluindo IRAS.

Compreender a disseminação das bactérias de interesse clínico nas superfícies bióticas e inertes em um ambiente hospitalar é importante para a vigilância em saúde no sentido de intensificar: (1) as metodologias de prevenção e controle desses agentes, bem como (2) ações que evitem o incremento desordenado de microrganismos resistentes. Desse modo, conforme observações decorrentes desse estudo, destaca-se a necessidade de adequada descontaminação das mãos dos profissionais de saúde e das superfícies abióticas em locais de assistência à saúde, visando reforço da biossegurança e mitigação da dispersão de patógenos (multi) resistentes e as IRAS decorrentes destes.

1. Ataee RA, Ataee MH, Tavana AM, Salesi M. Bacteriological aspects of hand washing: a key for health promotion and infections control. Int J Prev Med. 2017; 8:16.

2. Lozano C, Torres C. Update on antibiotic resistance in Gram-positive bacteria. Enferm Infecc Microbiol Clin. 2017; 35(1):2-8.

3. European Centre for Disease prevention and Control (ECDC). Healthcare-associated infections. 2016 [acesso em: 07 ago. 2019]. Disponível em: https://www.ecdc.europa.eu/en/heal thcare-associated-infections.

4. World Health Organization. Antimicrobial resistance Fact sheet. 2020 [acesso em: 07 ago. 2019]. Disponível em: https: / /www. who.int/newsroom/factsheets/detail/antimicrobialresistance.

5. Teixeira-Rodrigues A, Roque F, Falcão A, Figueiras A, Herdeiro MT. Understanding physician antibiotic prescribing behaviour: A systematic review of qualitative studies. Int J Antimicrob Agents. 2013; 41(3):20312.

\section{REFERÊNCIAS}


6. Koulenti D, Xu E, Song A, Mok IYS, Karageorgopoulos DE, Armaganidis A, et al. Emerging Treatment Options for Infections by Multidrug-Resistant Gram-Positive Microorganisms. Microorganisms. 2020; 8(2):191.

7. Van den Bogaard AE, Stobberingh EE. Epidemiology of resistance to antibiotics: Links between animals and humans. Int J Antimicrob Agents. 2000; 14(4):327-35.

8. Fletcher S. Understanding the contribution of environmental factors in the spread of antimicrobial resistance. Environ Health Prev Med. 2015; 20(4):243-52.

9. Becker K, Heilmann C, Peters G. Coagulase-negative staphylococci. Clin Microbiol Rev. 2014; 27(4):870-926.

10. Lakhundi S, Zhang K. Methicillinresistant Staphylococcus aureus: molecular characterization, evolution, and epidemiology. Clin Microbiol Rev. 2018; 31(4):e0002018.

11. McGuinness WA, Malachowa N, DeLeo FR. Vancomycin resistance in Staphylococcus aureus. Yale J Biol Med. 2017; 90(2):269-81.

12. Klevens RM, Edwards JR, Tenover FC, McDonald LC, Horan T, Gaynes R. Changes in the epidemiology of methicillin-resistant Staphylococcus aureus in intensive care units in US hospitals, 1992-2003. Clin Infect Dis. 2006; 42(3):389-91.

13. Teeraputon S, Santanirand $P$, Wongchai T, Songjang W, Lapsomthob N, Jaikrasun D, et al. Prevalence of methicillin resistance and Macrolides-LincosamidesStreptogramin B Resistance in Staphylococcus haemolyticus among clinical strains at Tertiary Care Hospital Thailand. New Microbes New Infect. 2017; 19:28-33.

14. Widerström M. Significance of Staphylococcus epidermidis in health care-associated infections, from contaminant to clinically relevant pathogen: this is a wake-up call! J Clin Microbiol. 2016; 54(7):16791681.

15. Rigatti F, Tizotti MK, Hörner R, Domingues VO, Martini R, Mayer LE, et al. Bacteremias por Staphylococcus coagulase negativos oxacilina resistentes em um hospital escola na cidade de Santa Maria, Estado do Rio Grande do Sul. Rev Soc Bras Med Trop. 2010; 43(6):686-690.

16. Pinheiro L, Brito $\mathrm{Cl}$, Oliveira $\mathrm{A}$, Pereira VC, Cunha MLRS. Staphylococcus epidermidis and Staphylococcus haemolyticus: detection of biofilm genes and biofilm formation in blood culture 
isolates from patients in a Brazilian teaching hospital. Diagn Microbiol Infect Dis. 2016; 86(1):11-14.

17. Rossi CC, Pereira MF, GiambiagideMarval $M$. Underrated Staphylococcus species and their role in antimicrobial resistance spreading. Genet Mol Biol. 2020; 43(1 suppl 2):e20190065.

18. Kim PH, Leopold SS. GustiloAnderson classification. Clin Orthop Relat Res. 2012; 470(11):3270-74.

19. Ozer TT. The rate of inducible MLSB resistance in the methicillin-resistant Staphylococci isolated from clinical samples. J Clin Lab Anal. 2016; 30(5):490-3.

20. Pérez-Trallero E, Montes $M$, Orden B, Tamayo E, García-Arenzana JM, Marimón JM. Phenotypic and genotypic characterization of Streptococcus pyogenes isolates displaying the MLSB phenotype of macrolide resistance in Spain, 1999 to 2005. Antimicrob Agents Chemother. 2007; 51(4):1228-33.

21. Saderi H, Emadi B, Owlia P. Phenotypic and genotypic study of macrolide, lincosamide and streptogramin B (MLSB) resistance in clinical isolates of Staphylococcus aureus in Tehran, Iran. Med Sci Monit. 2011; 17(2):BR48-BR53.
22. Méric G, Miragaia $M$, Been M, Yahara $\mathrm{K}$, Pascoe B, Mageiros L, et al. Ecological overlap and horizontal gene transfer in Staphylococcus aureus and Staphylococcus epidermidis. Genome Biol Evol. 2015; 7(5):1313-28.

23. Baddour LM, Wilson WR, Bayer AS, Fowler Júnior VG, Bolger AF, Levison $M E$, et al. Infective endocarditis: diagnosis, antimicrobial therapy, and management of complications: a statement for healthcare professionals from the Committee on Rheumatic Fever, Endocarditis, and Kawasaki Disease, Council on Cardiovascular Disease in the Young, and the Councils on Clinical Cardiology, Stroke, and Cardiovascular Surgery and Anesthesia, American Heart Association: endorsed by the Infectious Diseases Society of America. Circulation. 2005; 111(23):e394-434.

24. Edelsberg J, Weycker D, Barron R, Li $\mathrm{X}$, Wu H, Oster $\mathrm{G}$, et al. Prevalence of antibiotic resistance in US hospitals. Diagn Microbiol Infect Dis. 2014; 78(3):255-62.

25. Amberpet R, Sistla S, Parija SC, Thabah MM. Screening for intestinal colonization with Vancomycin 
Resistant Enterococci and associated risk factors among patients admitted to an Adult Intensive Care Unit of a large teaching hospital. J Clin Diagn Res. 2016; 10:DC06-9.

26. Sood S, Malhotra M, Das BK, Kapil A. Enterococcal infections \& antimicrobial resistance. Indian J Med Res. 2008; 128:111-21.

27. García-Solache M, Rice LB. The Enterococcus: a Model of adaptability to its environment. Clin Microbiol Rev. 2019; 32(2):e0005818.

28. Golob $M$, Pate $M$, Kušar $D$, Dermota $U$, Avberšek J, Papić $B$, et al. Antimicrobial resistance and virulence genes in Enterococcus faecium and Enterococcus faecalis from humans and retail red meat. Biomed Res Int. 2019; 2019:2815279.

29. Delpech G, Pourcel G, Schell C, De Luca M, Basualdo J, Bernstein J, et al. Antimicrobial resistance profiles of Enterococcus faecalis and Enterococcus faecium isolated from artisanal food of animal origin in Argentina. Foodborne Pathog Dis. 2012; 9(10):939-44.

30. Raza T, Ullah SR, Mehmood K, Andleeb S. Vancomycin resistant Enterococci: a brief review. J Pak Med Assoc. 2018; 68(5):768-72.

31. Torres C, Alonso CA, Ruiz-Ripa L, León-Sampedro R, Del Campo R, Coque TM. Antimicrobial resistance in Enterococcus spp. of animal origin. Microbiol Spectr. 2018; 6(4).

Financiamento: Agradecimentos à FAPEMAT pelo fomento ao projeto de pesquisa sob $n^{\circ} 0214392 / 2017$ do Edital Universal 042/2016 e pela bolsa de estudos conforme processo 0470038/2018.

Conflito de interesses: Os autores declaram não haver conflito de interesses.

\section{Participação dos autores:}

- Concepção: Machado AP, Valim MD, Corrêa LVA, Corrêa ER, Melo LR, Topanotti ATR, Atakiama VT, Pepato MA.

- Desenvolvimento: Machado AP, Valim MD, Corrêa LVA, Corrêa ER, Melo LR, Topanotti ATR, Atakiama VT, Pepato MA.

- Redação e revisão: Machado AP, Valim MD, Corrêa LVA, Corrêa ER, Melo LR, Topanotti ATR, Atakiama VT, Pepato MA.

Como citar este artigo: Machado AP, Valim MD, Corrêa LVA, Corrêa ER, Melo LR, Topanotti ATR, et al. Cocos Gram-positivos resistentes isolados de profissionais de saúde e de ambiente hospitalar em Cuiabá-MT. J Health NPEPS. 2021; 6(1):256-271. 